\title{
A Surgical Case of Giant Malignant Mesenchymoma in the Posterior Mediastinum That Recurred in the Bilateral Mediastinum
}

\author{
Shigeo Tanimura ${ }^{1,2}$, Yuji Saito ${ }^{1,2}$, Kazuo Honma ${ }^{3}$ and Kiyoshi Koizumi ${ }^{1}$ \\ ${ }^{1}$ Department of Biological Regulation and Regenerative Surgery, Graduate School of Medicine, Nippon Medical School \\ ${ }^{2}$ Department of Thoracic Surgery, Nippon Medical School Tama Nagayama Hospital \\ ${ }^{3}$ Department of Pulmonary Medicine, Sagamihara Kyodo Hospital, Kanagawa
}

\begin{abstract}
Malignant mesenchymoma is a soft-tissue tumor arising preferentially in the extremities and the retroperitoneum. We report a case of primary malignant mesenchymoma of the left side of the posterior mediastinum with local recurrence on both sides of the mediastinum. A 24-year-old woman was admitted to our hospital complaining of cough. Chest X-ray showed a giant mass in two-thirds of the left lung field, and computed tomography of the chest revealed a lobulated large mass with a fat-density area and a calcified spot in the mediastinum and left pleural space. Magnetic resonance of the chest demonstrated a large, solid mass consisting mainly of areas with the same intensity as fatty tissue and partly of areas of heterogeneous moderate intensity. The tumor was resected completely through a left posterolateral thoracotomy. The pathologic diagnosis was malignant mesenchymoma: well-differentiated liposarcoma with osteocartilagenous differentiation. The patient had local recurrence in both sides of the mediastinum 3 years and 6 months after surgery, and the recurrent tumors were completely resected again.
\end{abstract}

(J Nippon Med Sch 2008; 75: 212-215)

Key words: malignant mesenchymoma, mediastinal tumor, local recurrence

\section{Introduction}

Malignant mesenchymoma is a tumor consisting of two or more types of well-differentiated sarcoma components that rarely develops in the mediastinum. We treated a patient who had malignant mesenchymoma arising in the left posterior mediastinum, which recurred on both sides of the mediastinum 3 years and 6 months after resection and was treatable with further surgery.

\section{Case Report}

The patient was a 24-year-old woman who had severe cough starting in early May 2002 and visited the Respiratory Department of Sagamihara Kyoudo Hospital in July. A chest X-ray examination showed a shadow of a giant mass in the left side of the thorax (Fig. 1), and the patient was admitted for emergency treatment. Computed tomography (CT) and magnetic resonance imaging (MRI) of the chest suggested a giant mediastinal tumor (specifically, a teratoid tumor), and the patient was transferred to

Correspondence to Shigeo Tanimura, Department of Thoracic Surgery, Nippon Medical School Tama Nagayama Hospital, 1-1-7 nagayama, tama-shi, Tokyo 206-8512, Japan

E-mail: kogeka-tanimura@nms.ac.jp

Journal Website (http://www.nms.ac.jp/jnms/) 


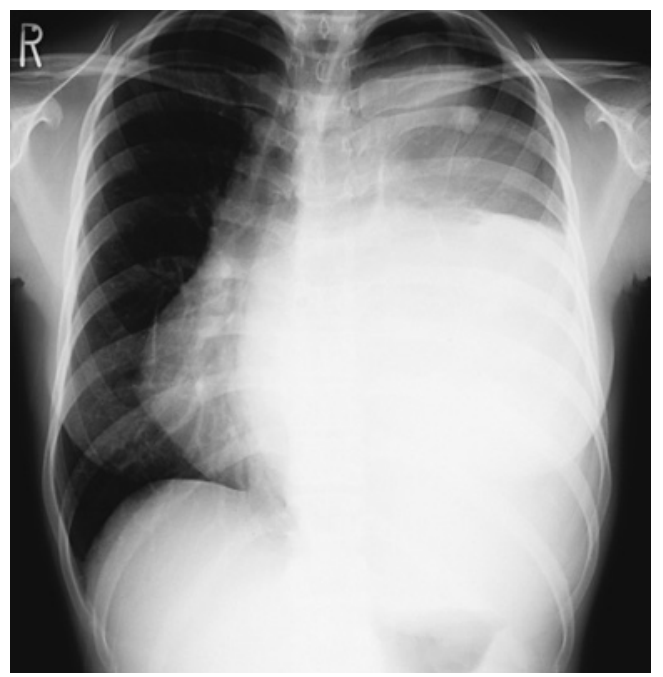

Fig. 1 Chest radiograph showing a giant mass occupying two-thirds of the left side of the thoracic cavity.

the Department of Thoracic Surgery at the end of July.

The physical findings on admission were height, $150 \mathrm{~cm}$; body weight, $50 \mathrm{~kg}$; body temperature, $37.2^{\circ} \mathrm{C}$; and blood pressure, $114 / 78 \mathrm{mmHg}$. Respiratory sounds of the left lung were markedly reduced on auscultation, but no rales were heard. There were no abnormalities on laboratory tests, except for a C-reactive protein level of $5.5 \mathrm{mg} / \mathrm{dL}$ and a white blood cell count of $6,800 / \mu \mathrm{L}$. Respiratory function tests could not be performed, but the results of arterial blood gas analysis (under normal pressure) were normal: $\mathrm{PaO}_{2}, 92$ torr; and $\mathrm{PaCO}_{2}, 38$ torr. Tumor marker findings were normal. On chest CT, a giant tumor with areas of mixed density corresponding to fatty tissue and calcification occupied two-thirds of the left side of the thoracic cavity which compressed the lung in an anterior and upward direction (Fig. 2). On MRI, the lung was partly visible in the upper anterior region of the field, but the tumor occupied most of the view, with various mixed fatty and calcified components. The diaphragm was compressed downward. On the basis of these findings, we concluded on the basis of the fatty tissue and calcification that the tumor that originated in the posterior mediastinum was a teratoid tumor.

At the end of July 2002, an operation was performed with the patient in the right lateral position and under general anesthesia, after the stability of circulatory dynamics and respiratory

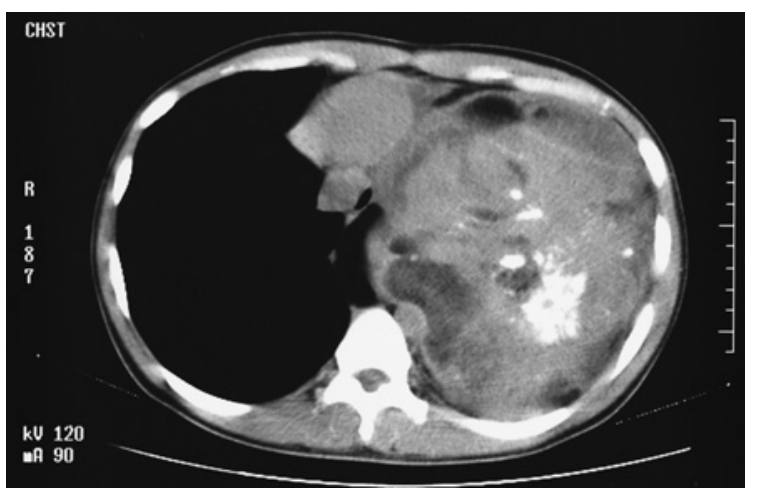

Fig. 2 Chest CT showing a giant tumor consisting of mixed regions with intensities consistent with fatty tissue and calcification occupied two-thirds of the left side of the thoracic cavity and compressed the lung in an anterior and upward direction.

conditions had been confirmed. A 25-cm S-curved skin incision was made in the left posterolateral region, and the thorax was opened at the 5 th and 9 th intercostal spaces. The tumor was located in the dorsal region of the thoracic cavity and was strongly and fibrously adherent to the lower lobe, pericardium, aorta, and diaphragm. The adhesion was detached at the tumor capsule, and the tumor was completely removed macroscopically. The operation time was 5 hours and 25 minutes, and the blood loss was $1,026 \mathrm{~mL}$. The tumor was $27 \times 19 \times$ $11 \mathrm{~cm}$ in size and weighed $3.2 \mathrm{~kg}$. The tumor consisted of nodular lesions of various size, with a mixture of yellowish, fat-like, hard, fibrous, and bonelike regions. Histopathological examination showed a well-differentiated region assumed to be lipoma; a region composed of atypical adipocytes, suggesting liposarcoma; and a fibrosarcoma-like region occupying most of the tumor (Fig. 3a). Bone formation and a chondrosarcoma-like region were also noted (Fig. 3b). On the basis of the above findings, the tumor was diagnosed as a malignant mesenchymoma.

Hypoventilation of the left lower lobe due to compression resolved immediately after surgery, and the lobe was almost completely dilated. The postoperative course was uneventful, and the patient was discharged on day 12 after surgery.

In February 2006, 3 years and 6 months after surgery, an abnormal shadow was noted on chest Xray examination during follow-up at the outpatient clinic, and the patient was re-admitted for thorough 


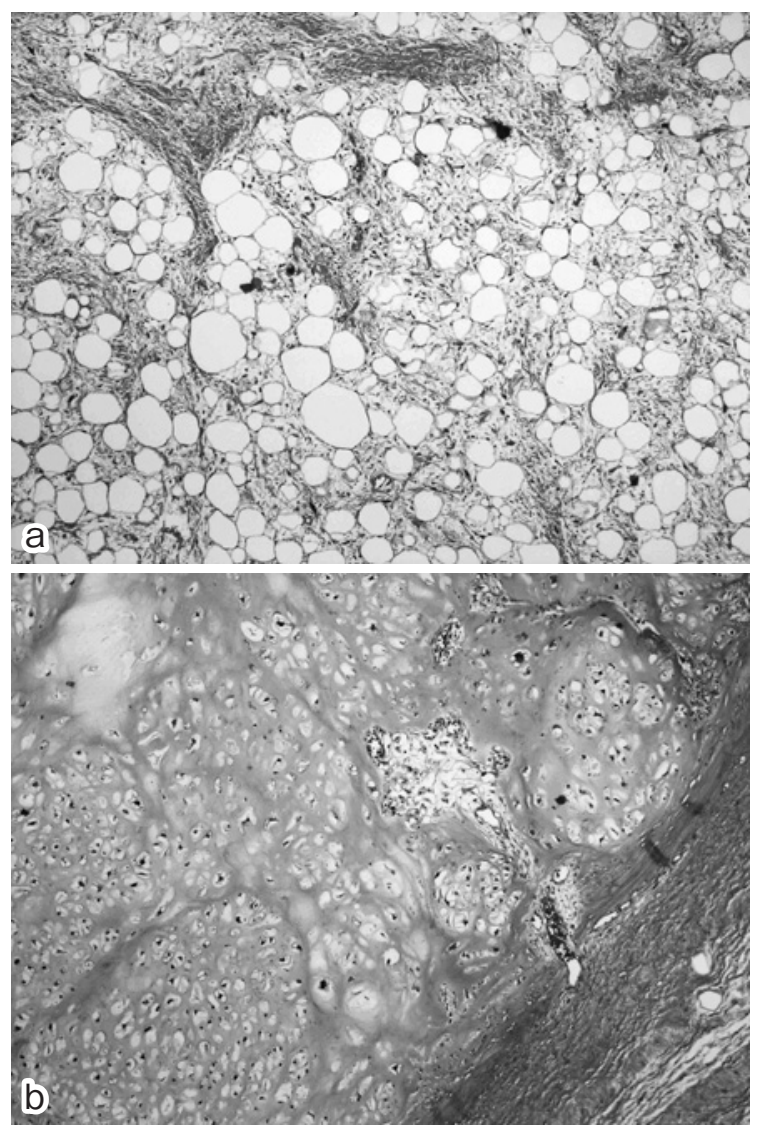

Fig. 3 Histopathologic examination showed regions of a) liposarcoma and b) chondrosarcoma.

examination and treatment. On chest CT, the tumor was found to have recurred on both sides of the mediastinum, in contact with the thoracic vertebrae (Fig. 4). Surgery was performed first for the recurrent tumor on the left side of the mediastinum. A left lateral incision was made, and the thorax was opened at the 7th intercostal space. No anterior adhesion in the thoracic cavity or severe adhesion around the tumor was noted; adhesion of the tumor to the lower lobe, esophagus, and aorta was slight; and no infiltration was noted. The adhesion was detached at the tumor capsule, and the tumor was completely excised. The operation time was 1 hour and 35 minutes, and the blood loss was $47 \mathrm{~mL}$. Histopathological examination showed that the tumor was mostly fibrosarcoma and partially chondrosarcoma.

Surgery for the tumor of the right side was performed 1 month later. A posterolateral incision was made on the right side, and the thorax was opened at the 6th intercostal space. No adhesion was present in the thoracic cavity, and the border of the tumor was clear without infiltration. The tumor was

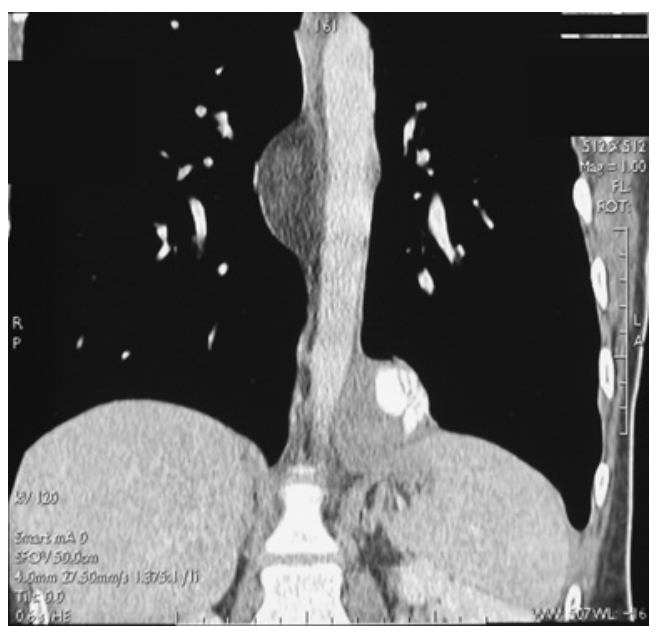

Fig. 4 Chest CT showing a 7 -cm tumor on the right side consisting mainly of a fatty component and in contact with the 9th thoracic vertebra; and a 4-cm partially calcified tumor on the left side between the 12th thoracic vertebra and the diaphragm.

detached in the capsular region and completely excised. The operation time was 1 hour and 45 minutes, and the blood loss was $20 \mathrm{~mL}$. Histopathological examination showed that the tumor was mostly liposarcoma and partially fibrosarcoma, but no osteosarcoma or chondrosarcoma component was present.

The tumor had not recurred by 1 year and 8 months after surgery.

\section{Discussion}

Stout reported the first case of soft-tissue malignant tumor composed of two or more types of mesenchymal cell-derived tumors as malignant mesenchymoma in $1948^{1}$. Malignant mesenchymoma frequently develops in the retroperitoneum and the soft tissue of the lower limbs. The development of such tumors in the heart, esophagus, liver, urinary bladder and larynx has also been reported, but development in the mediastinum is extremely $\operatorname{rare}^{2-7}$. Tumors have been reported in persons ranging in age from childhood to old age, but the incidence tends to be highest in persons 60 years or older ${ }^{3}$.

Regarding the imaging characteristics of malignant mesenchymoma, CT and MRI images reflect the histopathological heterogeneity of the tumor. Mixed areas show the density of lipoma or liposarcoma; differentiation into bone, cartilage and muscle; and fibrosarcoma-like components; and 
findings indicating the fusion of multiple solid nodules are considered to be characteristic ${ }^{8}$. Differentiation from malignant teratoid tumor is possible based on the absence of a cystic structure. Our case showed these imaging characteristics.

Histological diagnosis of the tumor is performed with Stout's definition. Neither undifferentiated sarcoma nor a fibrosarcoma-like region is counted as a component, and these are not essential elements for diagnosis of malignant mesenchymoma. Various histological findings have been noted and can roughly be divided into the following two types: 1) fibrosarcoma-like spindle cell components are often present, but myogenic sarcoma (rhabdomyoma, leiomyosarcoma) and liposarcoma are mixed; and 2) sarcoma components are apparently differentiated, such as rhabdomyoma and liposarcoma, and are accompanied by malignant cartilage and bone tissue components. In our patient, the tumor consisted mostly of fatty and fibrous components accompanied by atypia, and partial atypical cartilage and bone components were noted, suggesting classification into type 2 above. Diseases requiring histopathological differentiation include liposarcoma. In one reported case, well-differentiated liposarcoma developed in the mediastinum in the first episode and dedifferentiated upon recurrence in the mediastinum, with appearance of rhabdomyoma and osteosarcoma and chondrosarcoma components 5 . In such cases, differentiation from malignant mesenchymoma is extremely difficult.

Complete surgical excision is the principle treatment for malignant mesenchymoma, and chemotherapy and radiotherapy may be ineffective. In our patient, the tumor was strongly adherent to the left lower lobe, pericardium, chest wall, aorta, and diaphragm, but detachment of the layer assumed to be the capsule was possible, and complete tumor excision was performed macroscopically without the resection of other organs. The hypoventilated lower lobe returned to an almost original condition, and the patient recovered without postoperative complications, although the tumor recurred on both sides of the posterior mediastinum after 3 years and 6 months, perhaps because of local recurrence of microscopic residual tumor remaining in the mediastinal pleura.

A poor prognosis has been reported for malignant mesenchymoma. In 8 femoral and retroperitoneal cases described by Brady et $\mathrm{al}^{3}$, the 2- and 3-year survival rates were $75 \%$ and $37 \%$, respectively, and none of the patients survived for more than 5 years. Because the tumor recurred 3 years and 6 months after surgery in our patient, careful follow-up will be necessary.

Chemotherapy and radiotherapy were ineffective for the soft parts of the sarcoma, including the malignant mesenchymoma, but the efficacy of chemotherapy with doxorubicin plus ifosfamide and cyclophosphamide, vincristine, doxorubicin, and dacarbazine has recently been reported $^{9-11}$. Thus, a multidisciplinary approach including surgery, radiotherapy, and chemotherapy may be useful for these tumors.

\section{References}

1. Stout AP: Mesenchymoma, the mixed tumor of mesenchymal derivatives. Ann Surg 1948; 127: 278290.

2. Enzinger FM, Weiss SW: Malignant mesenchymoma. In Soft tissue tumors, $3^{\text {rd }}$ ed, 1995; pp 1087-1089, Mosby-Year Book, St Louis.

3. Brady MS, Perino G, Tallini G, Russo P, Woodruff JM: Malignant mesenchymoma. Cancer 1996; 77: 467-473.

4. Newman PL, Fletcher CDM : Malignant mesenchymoma. Am J Surg Pathol 1991; 15: 607-614.

5. Mukherji SK, Rojiami AM, Younathan CM: CT findings of retroperitoneal malignant mesenchymoma. Abdom Imaging 1994; 19: 82-83.

6. Misao T, Nakagawa J, Nakano H, et al: A case of resection of malignant mesenchymoma developed in the mediastinum. Haigan 2000; 40: 651-656.

7. Al-Kana R, Rangwala AF, Sills C, Rienzo A: Case report: malignant mesenchymoma of the mediastinum. New Jersey Medicine 1992; 89: 851855.

8. Hayama M, Nagahiro W, Aoe S, et al.: A case of welldifferentiated liposarcoma that recurred with dedifferentiation in the local region 11 years after resection. Jpn J Chest Surg 2001; 15: 521-527.

9. Glenn J, Kinsella T, Glatstein E, et al.: A randomized, prospective trial of adjuvant chemotherapy in adults with soft tissue sarcomas of the head and neck, breast, and trunk. Cancer 1985; 15: 1206-1214.

10. Issels RD, Schlemmer M: Current trials and new aspects in soft tissue sarcoma of adults. Cancer Chemother Phamacol 2002; 49 Suppl 1: S4-8.

11. Wall N, Starkhammar H: Chemotherapy of soft tissue sarcoma-a clinical evaluation of treatment over ten years. Acta Oncol 2003; 42: 55-61.

(Received, December 21, 2007)

(Accepted, March 31, 2008) 